\title{
Study on Fluid-Structure Interaction for the Multistage High Speed Centrifugal Pumps Rotors
}

\author{
Tian Yabin ${ }^{*}, 1$, Wang Jing $^{2}$ and Wang Liang ${ }^{1}$ \\ ${ }^{I}$ School of Civil Engineering and Architecture, Southwest University of Science and Technology, MianYang, Postcard: \\ 621010, China \\ ${ }^{2}$ Jinchuan Group Co., LTD., Jinchuan 737100, China
}

\begin{abstract}
In the designing process of high-speed multistage centrifugal pump, in addition to the hydraulic characteristics, the calculation problem of the axis of wet critical speed is the emphasis of concern. On the base of the finite element model of the "dry" state rotor dynamics, when the flow force play a individual role in physical separate discs and cylinder, resistance formula is derived. Establish the "wet" mode of motion equation of rotor disc and shaft section, then integrate the flow force into the motion equation of the whole system unit, we can find the fluid-solid coupling finite element model of the rotor dynamics. Write the calculation procedure with ANSYS APDL language, and the analysis and testing of fluidsolid coupling dynamics program to an actual high-speed multistage centrifugal pump rotor system is done.
\end{abstract}

Keywords: Fluid-structure interaction, multistage centrifugal pump, rotor dynamics.

\section{INTRODUCTION}

High-speed multistage centrifugal pump has been extensively applied in petroleum, chemical industry, electric power, steel and other important industry areas. As the important auxiliary of production device, with the increasing of single-machine capacity of large-scale production device pump units and the requirement of the operation reliability is stricter. Therefore, in the designing phase of multistage centrifugal pump, the rotor system analysis of dynamic properties is very important.

When the rotor system of multistage centrifugal pump is analysed, it is different from the general rotor system, that is to say in addition to the considering of the dynamic characteristics of pump shaft and the oil film bearing, gyro effect and the influence of a state fluid-solid coupling and other factors to the dynamic characteristics in the impeller working should also be considered.

We know that most of the rotor systems are surrounded by fluid, the effect of fluid to the rotor is obvious, and with the increasing of rotor movement parameters (such as speed), the effect of the rotor to the simulating subsystem dynamic characteristics gets bigger, and it will lead to wrong results if neglect it. Although some content of Fluid Structure Interaction was gradually added in ADINA [1], ANSTRAN [2], ANSYS [3] and other commercial software of relevant structural dynamics, because of the complexity and special characteristics of the rotor system, they are unable to fully meet the requirement of the rotor dynamics system. In this kind of situation, scholars both domestic and overseas did a lot of work. R. Fritz [4], J. Antunes [5-8], Sun Qiguo [9] did studies on the Dynamic coefficient when the fluid machinery soaked in the big gap circulation, and the leaching. eccentric rotor is similar to the dynamic characteristics of oil film bearing coefficient. This way is used to try to solve the fluidsolid coupling problem of pump and turbine rotor system. But compared with the oil film bearing, due to the rotor structures differ in thousands ways, and it is very complex, the oil film bearing coefficient of the big gap circulation structure dynamic coefficient has not been found in engineering practice. There are a few scholars [10] who use the way of the additional quality ideal fluid to consider some effect of fluid-solid coupling in specific rotor structure, but the viscous resistance is ignored. This paper shows our attempt of finding a new way to establish a rotor dynamics of finite element modelling which can consider the fluidsolid coupling problem comprehensively and help us solve the fluid-solid coupling problem of the rotor system.

\section{THE ESTABLISHMENT OF MATHEMATCAL MODEL}

\subsection{The Power of the Fluid to the Moving Objects}

\subsubsection{Discs Do Translational Vibration in the Plane Parallel to Itself in Fluid}

The analysis of the fluid-structure coupling dynamics shows that the discs of which the radius $R$ is put in fluid, the density is $\rho$, and the viscosity is $\eta$, when it does translational vibration in the plane parallels to itself with the radian frequency $\omega$, the fluid resistance is as follows

$$
F=-m_{A} \ddot{x}-c \dot{x}
$$


In the type, $m_{\omega}=3 \pi R^{3} \sqrt{\frac{\eta \rho}{2 \omega}}, c_{\omega}=3 \pi R\left(1+\frac{2 R}{3 \delta}\right)$

\subsubsection{Discs Does Corner Vibration Around Symmetry Axis of Their Own Plane in Fluid}

When discs does Corner vibration, the fluid resistance moment is as follows

$M_{0}=-i \ddot{d a} g_{d a} \dot{\theta}$

In the type, $i_{\omega}=\frac{3 \pi}{8} R^{+} \sqrt{\frac{\eta \rho}{2 \omega}}\left(1+\frac{2 R}{3 \delta}\right), g_{d a}=\frac{3 \pi}{8} \eta R^{3}\left(1+\frac{R}{\delta}\right)$

\subsubsection{The Cylinder Does the Translation Vibration} Perpendicular to the Axis in Fluid

The fluid resistance of the translation on the unit length is as follows:

$q=-m_{s a} \ddot{x}-c_{s a} \dot{x}$

In the type, $m_{\omega}=6 \pi R \sqrt{\frac{\eta \rho}{2 \omega}}\left(1+\frac{R}{3 \delta}\right), c_{s \omega}=6 \pi \eta\left(1+\frac{R}{\delta}\right)$

\subsubsection{Cylinder Does Corner Vibration Around the Line} Perpendicular to Symmetry Axis of its Own Plane in Fluid

When cylinder does corner vibration, it does the cylinder surface at the length of $\mathrm{ds}$, the resistance moment of fluid resistance to the rotation centre corner is as follows:

$d M_{0}=-\left(m_{s a} \ddot{\theta}+c_{s o s} \dot{\theta}\right) s^{2} d s$

In the type, $m_{s a}$ and $c_{s a}$ is similar to type(3), Above all of types $\delta=\sqrt{\frac{2 \eta}{\omega \rho}}$

\subsection{The Finite Element Model of Fluid-solid Coupling}

\subsubsection{The Equation of Motion Disc}

According to the type (1) and (2), the nodal forces caused by fluid strength in the disk:

$$
\left.\begin{array}{l}
Q_{1 d}^{F}=-M_{d d} \ddot{u}_{1 d}-C_{d t} \dot{u}_{1 d} \\
Q_{2 d}^{F}=-M_{d t} \ddot{u}_{2 d}-C_{d d} \dot{u}_{2 d}
\end{array}\right\}
$$

$$
\text { In the type, } M_{d a}=\left(\begin{array}{cc}
m_{d a} & o \\
0 & i_{d a}
\end{array}\right), C_{d a}=\left(\begin{array}{cc}
c_{d a} & 0 \\
0 & g_{d a}
\end{array}\right)
$$
fluid

The equation of motion of the disc considering the role of

$$
\left.\begin{array}{l}
M_{d} \ddot{u}_{1 d}+G_{d} \dot{u}_{2 d}=Q_{1 d}+Q_{1 d}^{F} \\
M_{d} \ddot{u}_{2 d}-G_{d d} \dot{u}_{1 d}=Q_{2 d}+Q_{2 d}^{F}
\end{array}\right\}
$$

Take the equation (5) into (6) and rearranging the fluidsolid coupling equation of motion of the disc :

$\left.\begin{array}{l}M_{d} \ddot{u}_{1 d}+G_{d} \dot{u}_{2 d}+C_{d}^{*} \dot{u}_{1 d}=Q_{1 d} \\ M_{d} \ddot{u}_{2 d}-G_{d d} \dot{u}_{1 d}+C_{d}^{*} \dot{u}_{2 d}=Q_{2 d}\end{array}\right\}$
In the type, $M_{d}^{*}=M_{d}+M_{d}, C_{d}^{*}=C_{d \alpha}$

\subsubsection{The Equation of Motion of Shaft Unit}

The nodal forces caused by fluid resistance in the shaft unit. According to the principle of virtual work and notes $x=[N]\left\{U_{1 s}\right\}$

$\left\{\delta U_{1 s}\right\}^{T} Q_{1 s}^{F}=\int \delta x d F=\left\{\delta U_{1 s}\right\}^{T} \int[N]^{T} d F$

Thus

$Q_{1 s}^{F}=\int[N]^{T} d F$

And notes $d F=q d s$

$Q_{1 s}^{F}=-M_{s i s}^{F} \ddot{u}_{1 s}-C_{s}^{F} \dot{u}_{1 s}-K_{s}^{F} u_{1 s}$

In the type, $M_{x s}^{F}=\int_{0}^{l} m_{s}[N]^{T} N d s, \quad C_{s}^{F}=\int_{0}^{1} c_{s}[N]^{r} N d s$, $K_{s a}^{F}=\int_{s a}^{1} k_{s a}[N]^{T} N d s$

Obtained by the symmetry

$Q_{2 s}^{F}=-M_{s s}^{F} \ddot{u}_{2 s}-C_{s s}^{F} \dot{u}_{2 s}-K_{s i}^{F} u_{2,}$

The nodal forces caused by the fluid drag torque in the shaft unit. According to the principle of virtual work and notes $\theta=N^{\prime} u_{1 s}$

$\left\{\delta u_{1 s}\right\}^{T} Q_{1 s}^{M}=\int \delta \theta^{T} d M_{0}=\left\{\delta u_{1 s}\right\}^{T} \int\left[N^{\prime}\right]^{T} d M_{0}$

Thus

$Q_{1 s}^{M \prime}=\int\left[N^{\prime}\right]^{r} d M_{0}$

Take the type (12) into (13)

$Q_{1 s}^{M}=-M_{s a}^{M} \ddot{u}_{\mathrm{s} s}-C_{s}^{M} \dot{u}_{\mathrm{ts}}-K_{s a}^{M} u$

In the type, $M_{s a}^{M}=\int_{0}^{1} m_{s e}\left[N^{\prime}\right]^{r} N^{\prime} s^{2} d s, C_{s a}^{M}=\int_{0}^{1} c_{s a}\left[N^{\prime}\right]^{r} N^{\prime} s^{2} d s$, $K_{s e s}^{\prime \prime}=\int_{0}^{1} k_{s e s}\left[N^{\prime}\right]^{r} N^{\prime} s^{2} d s$

Obtained by the symmetry

$Q_{2 s}^{M}=-M_{s i s}^{M} \ddot{u}_{2 s}-C_{s w}^{M} \dot{u}_{2 s}-K_{s i s}^{M} u_{2 s}$

the shaft unit equations of motion considering the role of the fluid.

$\left.\begin{array}{l}M \ddot{u}_{1 s}+G \dot{u}_{2 s}+K u_{1 s}=Q_{1 s}+Q_{1 s}^{F}+Q_{1 s}^{M} \\ M \ddot{u}_{2 s}-G \dot{u}_{1 s}+K u_{2 s}=Q_{2 s}+Q_{1 s}^{F}+Q_{2 s}^{M}\end{array}\right\}$

Take the equation (9), (10), (13), (14) into (16), and rearranging.

$\left.\begin{array}{c}M \ddot{u}_{1 s}+G \dot{u}_{2 s}+C_{s} \dot{u}_{1 s}+K_{s} u_{1 s}=Q_{1 s} \\ M \ddot{u}_{2 s}-G \dot{u}_{1 s}+C_{s} \dot{u}_{2 s}+K_{s} u_{2 s}=Q_{2 s}\end{array}\right\}$

In the type, $M_{s}^{*}=M_{s}+M_{s}^{F}+M_{s}^{M}$, $C_{s}^{*}=C_{s a}^{F}+C_{s a}^{M}$, 
$K_{s}^{*}=K_{s a}^{F}+K_{s}^{M}$

\subsubsection{The Equations of Motion of the Overall System}

The motion equations of the overall system considering the role of fluid should have the following form:

$\left.\begin{array}{l}M_{1}^{\prime} \ddot{U}_{1}+C_{1}^{*} \dot{U}_{1}+G_{1} \dot{U}_{2}+K_{1}^{*} U_{1}=Q_{1} \\ M_{1} \ddot{U}_{2}-G_{1} \dot{U}_{1}+C_{1}^{*} \dot{U}_{2}+K_{1}^{*} U_{2}=Q_{2}\end{array}\right\}$

Or $M \ddot{U}+C^{\prime \prime} \dot{U}+K^{\prime} U=Q$

In the type, $M^{*}=\left(\begin{array}{cc}M_{+} & 0 \\ 0 & M_{+}^{*}\end{array}\right)$ is the system inertia matrix as a whole considering the role of fluid, $C^{*}=\left(\begin{array}{cc}C_{1}^{*} & G_{1} \\ -G_{1} & C_{1}^{*}\end{array}\right)$ Is the damping matrix as a whole considering the role of fluid, $K=\left(\begin{array}{cc}K_{1} & 0 \\ 0 & K_{-}\end{array}\right)$Is the stiffness matrix as a whole considering the role of fluid. The shaft unit inertia matrix $M$ and disc cell inertia matrix $M$ format $M_{\text {, }}$, the shaft unit damping matrix $C$ and the disc unit damping matrix $C$ format $c$, the shaft unit element stiffness matrix $K$ and $\mathrm{s}$ the disc stiffness matrix $K$ format $K$.

\section{ANALYSIS OF THE ROTOR DYNAMICS}

The "dry" and "wet" critical speed of the rotor has been proposed in this paper. The "dry" is the rotor critical speed which does not include the impact of the fluid force, but the "wet" does.

For a high speed centrifugal pump of 10 stage, of which rated speed is $7500 \mathrm{r} / \mathrm{min}$, shaft diameter is $85 \mathrm{~mm}$, impeller is $215 \mathrm{~mm}$, chief of the spindle is about $2353 \mathrm{~mm}$ and diameter of the support is $70 \mathrm{~mm}$. Properties of material: spindle material is stainless steel $\mathrm{H} 410$ ( $1 \mathrm{Cr} 13)$, ratio of Poisson is 0.3 , density is $7850 \mathrm{Kg} / \mathrm{m}^{3}$, the density of young's modulus is $200 \mathrm{GPa}$. Strength the calculation program and analyze the system dynamic characteristics of the multistage centrifugal pump rotor by the parametric designing language (APDL) which is contained in ANSYS software. Because it is a high speed multistage centrifugal pump, the effect factors of gyro is written in programming, and the first threeorder natural frequency calculation results is shown as Table 1.

Table 1. The inherent frequency of high-speed multistage centrifugal pump.

\begin{tabular}{|c|c|c|}
\hline & Dry State & Wet State \\
\hline \hline first-order & 97.47 & 221.85 \\
\hline second-order & 268.39 & 290.31 \\
\hline third-order & 292.18 & 330.40 \\
\hline
\end{tabular}

(a) First-order

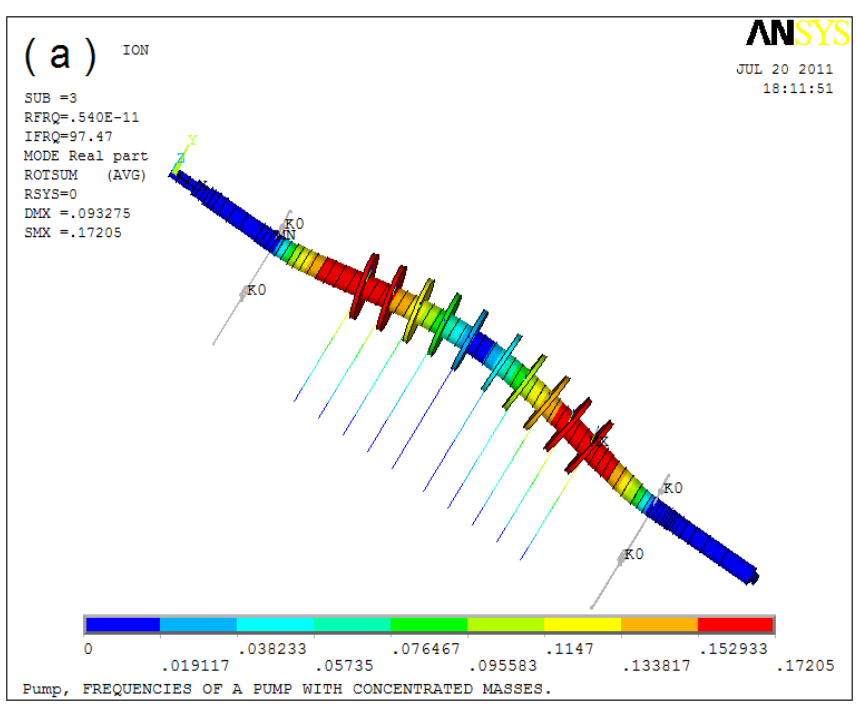

(b) Second-order

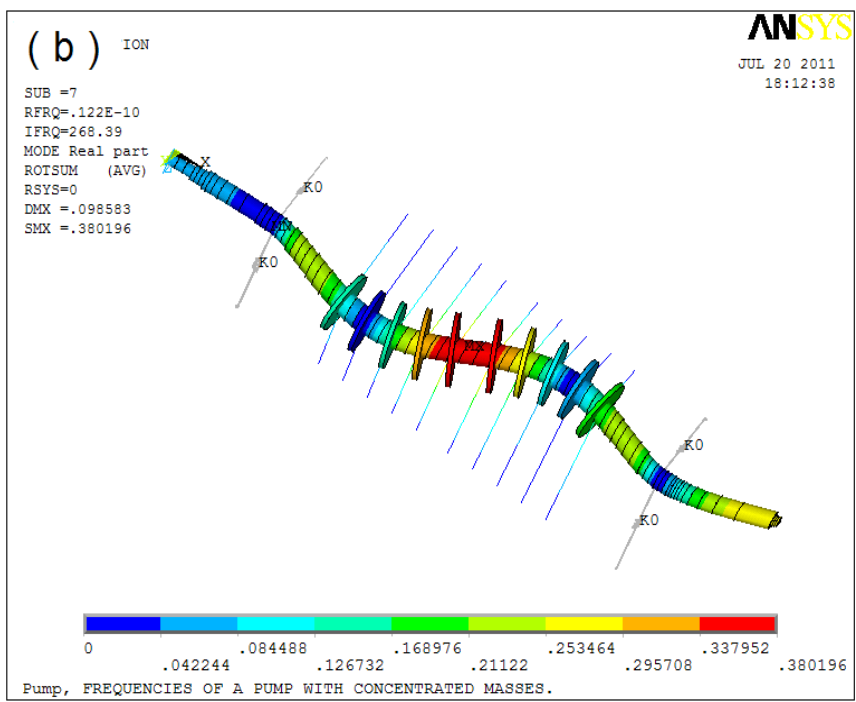

(c) Third-order

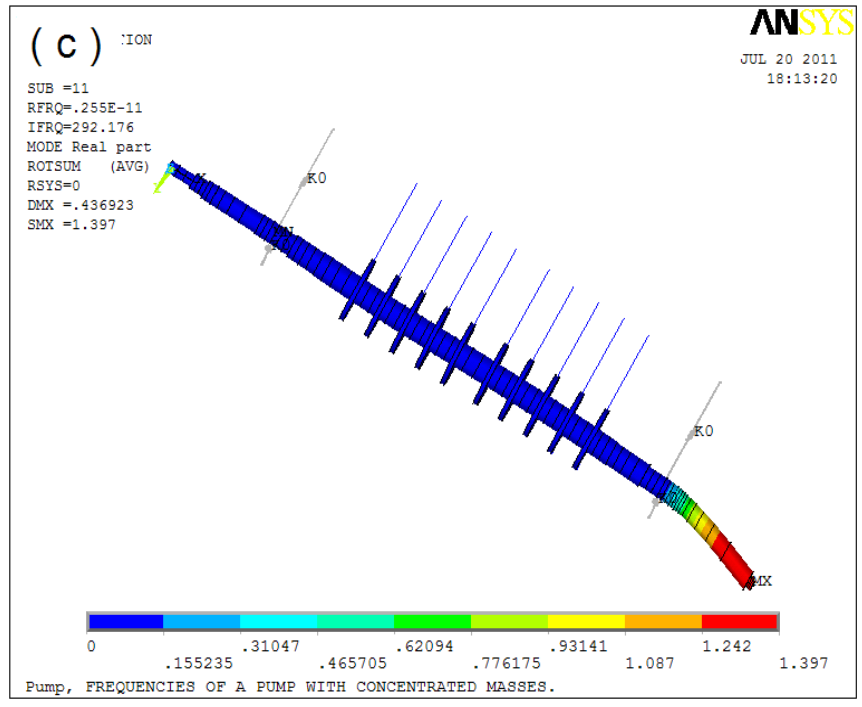

Fig. (1). The first three-order vibration type of dry state. 
(a) First-order

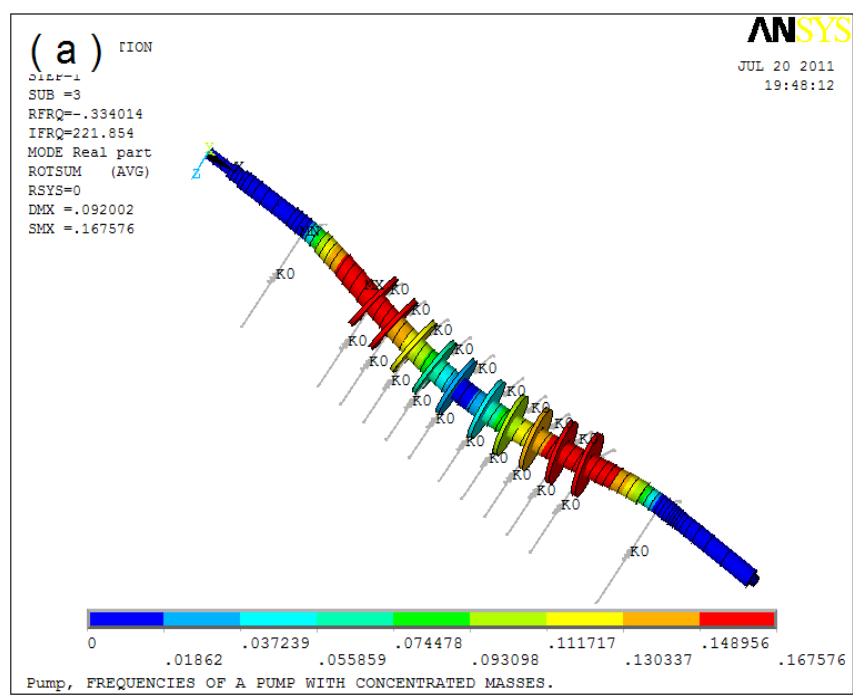

(b) Second-order

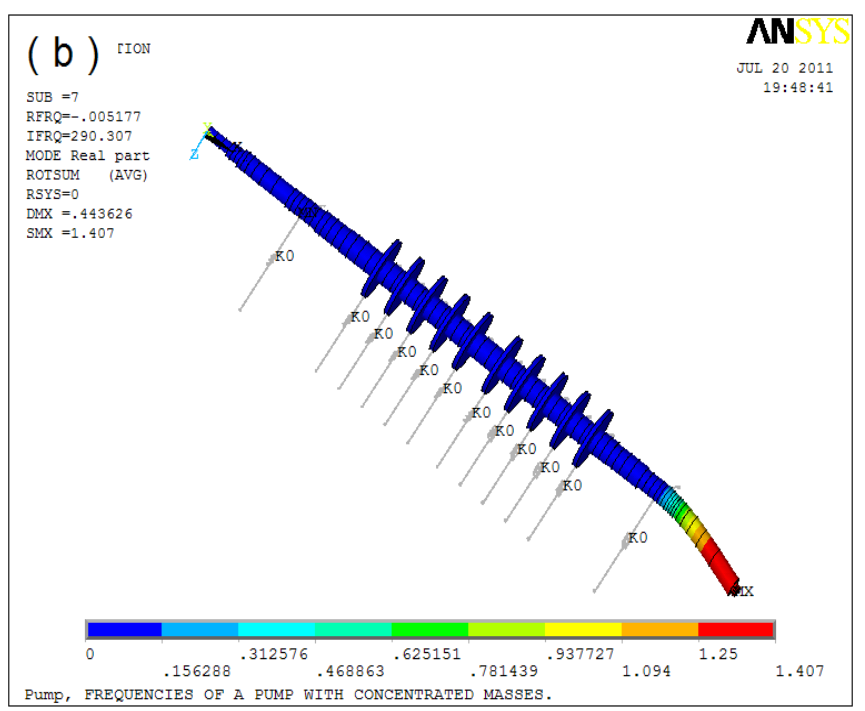

(c) Third-order

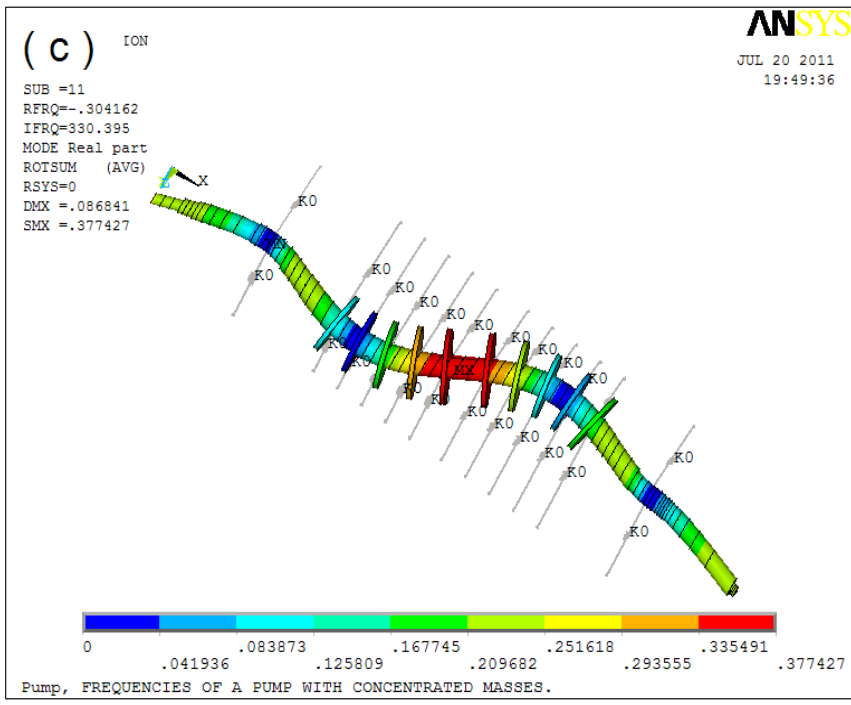

Fig. (2). The first three-order vibration type of wet state.
The result shows that critical speed of first order "wet" is obviously higher than that of the first order "dry" in the pump, and it is two times more than one order "dry" critical speed. The result shows that working speed of pump is lower than and away from the one order critical speed in the case of considering the fluid-solid coupling dynamic characteristics and it works in the range of safe speed. Figs. $(\mathbf{1}, \mathbf{2})$ shows that when fluid has effect, not only the natural frequency of the rotor, but also the mode of vibration changed. Action of Fluid to the impeller decreases imbalance response of the rotor, and it stabilized the rotor.

\section{CONCLUSION}

In the process of designing high-speed multistage centrifugal pump, flow has a significant effect to the dynamic performance of pump rotor.

(1) Integrate the role of fluid into the motion equation of the whole system to get fluid-solid coupling finite element model of the rotor dynamics. Doing dynamics analysis of a real high speed multistage centrifugal pump rotor system with the computer program of the finite element model, we can verify the theory that fluid has an effect on dynamics performance of transversal subsystem, and get conclusions by qualitative analysis.

(2) There are many differences of the critical speed between the state of dry and wet in multistage centrifugal pump, so the effect of fluid-solid coupling to dynamic characteristics must be considered when we design the calculation.

(3) The dynamics analysis of high speed multistage centrifugal pump rotor shows that, the effect of fluid to impeller will improve the stability of the rotor.

\section{CONFLICT OF INTEREST}

The authors confirm that this article content has no conflict of interest.

\section{ACKNOWLEDGEMENTS}

Project (13zx7145) supported by Research Foundation for PHD of Southwest University of Science and Technology (13zx7145)

\section{REFERENCES}

[1] H. Zhang, X.L. Zhang, and S. H. Ji, "Recent development of fluidstructure interaction capabilities in ADINA system," Computers and Structures, vol.81, no.8211, pp. 1071-1085, 2003.

[2] D. Thompson, C. Haley, and J. Ayres, "Development and application of NASTRAN structural optimization solution sequence within the BAE ECLIPSE system," Aeronautical Journal, vol.103, no.1026, pp. 399-403, 1999.

[3] J. F. Sigrist, C. Laine, and B. Peseux, "ANSYS computation of fluid-structure interaction: Numerical and experimental analysis of an elastic plate in contact with a compressible heavy fluid," Journal De Physique IV, vol.12, no. PR11, pp. 137-144, 2002.

[4] R. Fritz, "The effects of an annular fluid on the vibrations of along rotor: Part1-Theory ASME," Journal of Basic Engineering, no.92, pp. 923-929, 1970

[5] J. Antunes, F. Axisa, and T. Grunenwald, "Dynamics of rotors immersed in eccentric annular flow. Part1: Theory," Journal of Fluids and Structures, no.10, pp. 893-918, 1996.

[6] J. Antunes, J. Mendes, M. Moreira, and T. Grunenwald, "A theoretical model for nonlinear planar motions of rotors under fluid 
confinement," Journal of Fluids and Structures, no.13, pp. 103126, 1999.

[7] M. Moreira, J. Antunes, and H. Pina, "A theoretical model for nonlinear orbital motions of rotors under fluid confinement," Journal of Fluids and Structures, no.14, pp. 635-668, 2000.

[8] M. Moreira, J. Antunes, and H. Pina, "An improved linear model for rotors subject to dissipative annular flows," Journal of Fluids and Structures, no.17, pp. 813-832, 2003.
[9] Q. Sun, L. Yu, "Study of Dynamic Characteristics for Fluid Machine Rotor Immersed in Annular Liquid Flow," Power Engineering, vol. 20, no.5, pp. 906-910, 2000.

[10] C. Du, X. Yao, and Q. Chen, "Study on Fluid-solid Coupling Dynamic Characteristics for the Component of Hydraulic Turbines," Large Electric Machine and Hydraulic Turbine, no.6, pp. 47-52, 2001

(C) Yabin et al.; Licensee Bentham Open.

This is an open access article licensed under the terms of the Creative Commons Attribution Non-Commercial License (http://creativecommons.org/licenses/by-nc/3.0/) which permits unrestricted, non-commercial use, distribution and reproduction in any medium, provided the work is properly cited. 